\title{
Endoscopic Resection Combined with the Cryoballoon Focal Ablation system in the Porcine Normal Esophagus: A Preclinical Study
}

Hironori Sunakawa

Department of Gastroenterology and Endoscopy, National Cancer Center Hospital East, Kashiwa

\section{Yusuke Yoda}

Department of Gastroenterology and Endoscopy, National Cancer Center Hospital East, Kashiwa Nobuyoshi Takeshita

Department of Colorectal Surgery, National Cancer Center Hospital East, Kashiwa

\section{Hiro Hasegawa}

Department of Colorectal Surgery, National Cancer Center Hospital East, Kashiwa

\section{Kenji Takashima}

Department of Gastroenterology and Endoscopy, National Cancer Center Hospital East, Kashiwa

\section{Tomohiro Kadota}

Department of Gastroenterology and Endoscopy, National Cancer Center Hospital East, Kashiwa

\section{Takeo Fujita}

Division of Esophageal Surgery, National Cancer Center Hospital East, Kashiwa

\section{Tetsuo Akimoto}

Department of Radiation Oncology and Particle Therapy, National Cancer Center Hospital East, Kashiwa, Chiba

\section{Satoshi Fujii}

Division of Pathology, Exploratory Oncology Research \& Clinical Trial Center, National Cancer Center Tomonori Yano ( $\nabla$ toyano@east.ncc.go.jp )

Department of Gastroenterology and Endoscopy, National Cancer Center Hospital East, Kashiwa

\section{Research Article}

Keywords: combination treatment, cryoablation, endoscopic resection, esophageal neoplastic tissue

Posted Date: February 16th, 2021

DOl: https://doi.org/10.21203/rs.3.rs-196161/v1

License: (a) (i) This work is licensed under a Creative Commons Attribution 4.0 International License. Read Full License 


\section{Abstract}

Background: The Cryoballoon focal ablation system (CbFAS) for dysplastic Barrett's esophagus is simple, time-saving and has high therapeutic efficacy. This study aimed to evaluate the technical feasibility and tissue damage with combination therapy of endoscopic resection (ER) and CbFAS in porcine models.

Methods: Three pigs (A, B, and C) were included, and all ER procedures were performed by endoscopic mucosal resection using the Cap method (EMR). Combination therapy for each pig was performed as follows: (a) CbFAS was performed for a post-EMR mucosal defect for Pig A; (b) CbFAS for post-EMR scar for Pig B, and (c) EMR for post-CbFAS scar for Pig C. All pigs were euthanized at 32 days after the initial procedure, and the tissue damage was evaluated.

Results: All endoscopic procedures were followed as scheduled. None of the subjects experienced anorexia, rapid weight loss, bleeding, and perforation during the observation period. They were euthanized at 32 days after the initial endoscopic procedure. On histological assessment, there was little difference between the tissue that was treated with CbFAS alone and that treated with CbFAS in combination with ER.

Conclusion: Combination therapy with ER and CbFAS can be technically feasible, and its outcome was not significantly different from CbFAS alone in terms of tissue damage.

\section{Introduction}

Endoscopic resection (ER), including endoscopic mucosal resection (EMR) and endoscopic submucosal dissection (ESD), is widely accepted as a minimally invasive treatment for superficial esophageal squamous cell carcinoma (SESCC) [1]. Although there are cases of patients undergoing curative resection with ER, there exists a risk for developing multiple, metachronous or recurrent SESCC in the preserved segment of the esophagus [2]. Furthermore, these SESCC lesions may sometimes develop near the ER scar and pose challenges to ER because of postoperative submucosal fibrosis due to the previous ER. Another concern is that patients who have been treated with ER especially for a large SESCC or with repeated ER for multiple SESCC have an increased risk for esophageal strictures $[3,4]$.

The Cryoballoon focal ablation system (CbFAS; C2 Cryoballoon, HOYA Pentax Medical, Japan) has increasingly received attention as a novel device for the ablation of esophageal neoplastic tissue. Preliminary clinical studies in patients with Barrett's esophagus have shown that CbFAS is a simple, safe, and effective procedure for the removal of dysplastic Barrett's esophagus and esophageal squamous cell neoplasia [5-7]. Cryoablation works by making a cold ablations injury to the cells in the tissue while preserving the collagen matrix architecture. It differs from tissue-heating ablations such as radiofrequency ablation (RFA), which sometimes develop the fibrosis or stricture of esophageal lumen. Therefore, cryoablation is expected to have the potential to facilitate deeper ablation with lower stricture rates [8-10]. Thus, CbFAS may be a promising therapeutic option in combination with ER for lesions 
having a risk of esophageal stenosis due to ER or technical difficulty of ER. However, there is limited data on combination therapy with ER and CbFAS for SESCCs.

This study was conducted to evaluate the feasibility and safety of procedures and pathological tissue damage in combination therapy with ER and CbFAS in a porcine model.

\section{Methods}

\section{Experimental animals and study protocol}

This study was conducted at the National Cancer Center Hospital East, Kashiwa, Japan. The study protocol was approved by the Animal Experiment Committee in National Cancer Center JapanK18-024, 2018/12/27), and all animal experiments were conducted in accordance with the Institutional guidelines and the ARRIVE guidelines (http://www.nc3rs.org.uk/page.asp?id=1357). We included three female pigs (weight 40-45 kg). The study protocol was designed to minimize pain or discomfort to the animals. To evaluate the feasibility of combination therapy in the various situation, we created four lesions on each of the three porcine normal esophagi using a DualKnife ${ }^{\mathrm{TM}}$ (Olympus, Tokyo, Japan). We employed three combination therapy strategies: (a) simultaneous procedure of EMR and CbFAS for Pig A, (b) CbFAS for post-EMR scar for Pig B, and (c) ER for post-CbFAS scar for Pig C. All EMR procedures were performed through EMR using the Cap method (EMR).

\section{Study procedures}

In vivo porcine specimens were obtained under general anesthesia: sedation was induced with intramuscular midazolam (1 mg/kg) and ketamine $(15 \mathrm{mg} / \mathrm{kg})$, and anesthesia was induced and maintained with propofol ( $3 \mathrm{mg} / \mathrm{kg}$ initially, and $6 \mathrm{mg} / \mathrm{kg} / \mathrm{h}$, respectively). The details of procedural protocol in each pig were as follows. In Pig A, we simultaneously used EMR and CbFAS (ER + CbFAS; the CbFAS was done immediately after EMR for a post-EMR mucosal defect), wherein EMR + CbFAS were undertaken at two lesions on Day 1, and at another two lesions on Day 28 (Fig. 1) (Supplementary Video). In Pig B, we conducted CbFAS for a post-EMR scar; the EMR was done for two lesions on Day 1, and the CbFAS was carried out on two lesions of EMR scarring as well as at two lesions with normal mucosa on Day 28 after the EMR (Fig. 2). In Pig C, we conducted EMR for post-CbFAS scarring; CbFAS was done for two lesions on Day 1, and EMR was carried out for two lesions on the post-CbFAS scarring as well as for two normal lesions on Day 28. All endoscopic procedures were undertaken as scheduled by three surgeons. (HS, YY. TY)

\section{Cryoablation}

We used the CryoBalloon focal ablation system (CbFAS; C2 Cryoballoon, HOYA Pentax Medical, Japan) in this study. The CbFAS comprises a portable hand-held Controller, a catheter with a self-sizing balloon with 
a spray hole located on a diffuser, foot pedal for adjusting the balloon and the diffuser, and a single-use cartridge of liquid nitrous oxide. The distal tip of the catheter (diameter $3.6 \mathrm{~mm}$ ) is advanced through a PENTAX EG34-i10 therapeutic endoscope channel (HOYA Pentax Medical, Japan) and the proximal end is connected to the Controller to operate the catheter with the foot pedal. From the cartridge, which is also connected to the Controller, liquid nitrous oxide $\left(-85^{\circ} \mathrm{C}\right)$ is released through the catheter. By rotating the diffuser clockwise or counterclockwise, the spray hole can be directed to the targeted area. The balloon probe is placed in contact with the tissue wall of the target region, and cryogenic fluid is sprayed while visualizing the target site through the balloon. A single application created an ice patch of approximately $2 \mathrm{~cm}^{2}$ on the targeted mucosa. Ablations of 8 seconds durations were performed in this study.

\section{Endoscopic mucosal resection using a cap-fitted endoscope}

The EMR technique requires a specialized transparent cap that is fitted to the tip of an endoscope. Saline is injected into the submucosa. The crescent-shaped snare (Olympus, Tokyo, Japan) is then pre-looped into the groove of the rim of the cap. Then, the lesion is suctioned with medium to high vacuum into the cap. After the endoscopist strangulates the lesion by closing the snare, electrosurgical current is used to resect the lesion.

\section{Outcomes}

The primary outcome was to evaluate the feasibility of combination treatment. We evaluated the technical success (defined treatment completion as intended) and device malfunction (defined as any failure in CbFAS components requiring device replacement). In addition, the difficulty of each process in all procedures, including injection of saline into the submucosa, lifting the mucosa, and snaring and cutting in ER, and stabilizing the balloon and ablation in CbFAS, was recorded. The degree of difficulty was classified into three levels: easy, moderate, and difficult. Easy was defined by the procedure being performed without any problems, and difficult as instances when the procedure had failed several times and needed to be repeated, or when the treatment was not performed as planned. Moderate was defined as being intermediate in terms of ease and difficulty. In addition, all procedures were evaluated as easy or moderate in the technical success case. The secondary outcome was safety. We evaluated bleeding and perforation during treatment and esophageal stenosis, weight loss/gain, and anorexia during this study period.

\section{Histopathological analysis}

All animals were euthanized by intravenous injection of potassium chloride on days 32 after the initial procedure, and tissue specimens of EMR were harvested for histopathological examination. Histopathological outcomes were evaluated on the basis of tissue damage on days 5 and 32 after 
treatment. Tissue sections were prepared from samples of the ablated areas and EMR specimens. All specimens were fixed in formalin (10\%), embedded in paraffin, and stained with hematoxylin and eosin. Slices of the specimens were evaluated for depth of tissue damage and tissue finding caused by ablation damage in the esophageal wall. All specimens were assessed by a gastrointestinal pathologist.

\section{Results}

\section{Feasibility}

The procedure was technically successful in all combination therapy strategies. Technical difficulty and procedure success rate of each treatment strategy are shown in Table 1. In CbFAS even for EMR scars, the stability of balloon was achieved easily and there was no technical difficulty. In EMR for CbFAS scars, lifting with submucosal injection was not smooth as compared to that in the normal area, but the snaring and cutting was technically easy.

Table 1

Technical feasibility of combined ER and CbFAS

\begin{tabular}{|lll|}
\hline EMR vs EMR for CbFAS scar & & \\
\hline Injection to submucosa & EMR & EMR for CbFAS scar \\
\hline Mucosal lifting & Easy & Moderate \\
\hline Snaring and cutting & Easy & Easy \\
\hline Procedure success rate & $2 / 2$ & $2 / 2$ \\
\hline CbFAS vs CbFAS for EMR scar & & \\
\hline & CbFAS & CbFAS for EMR scar \\
\hline Stability of balloon & Easy & Easy \\
\hline Ablation & Easy & Easy \\
\hline Procedure success rate & $2 / 2$ & $2 / 2$ \\
\hline EMR, endoscopic mucosal resection; CbFAS, Cryoballoon focal ablation system
\end{tabular}

\section{Safety}

There was no treatment-related complication such as stenosis (Fig. 3). No device malfunction occurred in this study. None of the study animals experienced anorexia, weight loss, bleeding, or perforation during 


\section{Histological analysis}

Tissue damage of EMR after CbFAS specimens showed only mild fibrosis in the lamina propria mucosae compared to EMR specimens for normal areas. Tissue damage of treatment in pigs $A$ and $B$ are shown in Table 2. There was no difference in tissue damage between CbFAS alone and CbFAS combined with ER. Although the tissue damage of EMR+CbFAS spread all layers of the esophageal wall at 4 days after treatment, only fibrosis was observed in the submucosa at 32 days after treatment. (Fig. 4)

Table 2

Pathological evaluation of treatment in pigs $A$ and $B$

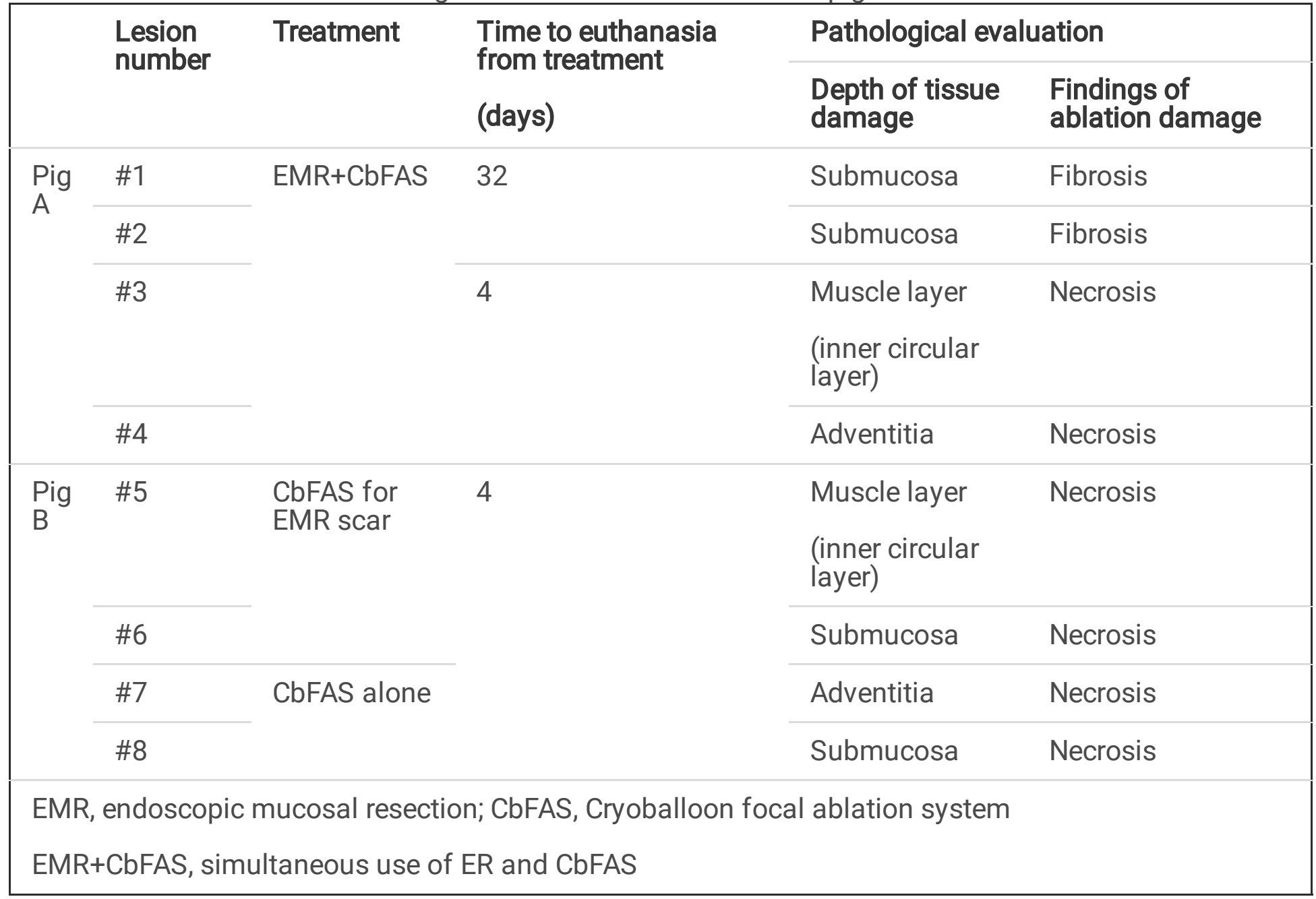

\section{Discussion}

In this study, we first assessed the technical feasibility and tissue damage of combined ER and CbFAS in an animal model. In the ER to the area after CbFAS, the ER procedure was successful even in cases with some unsteady flow and mucosal swelling during the saline injection into submucosa at the site of the 
minor scar after the CbFAS. Moreover, there were no major histological differences between the tissue of with ER followed by CbFAS or CbFAS alone. These results indicated that the combination of ER and CbFAS is feasible and is a promising treatment strategy for esophageal neoplasms.

We could comparatively evaluate the tissue damage due to EMR+CbFAS (simultaneous ER and CbFAS) between the acute and delayed phase in the same subject. The difference of findings was valuable because there have been only a few studies evaluating this method whereby a CbFAS for mucosal defects is performed immediately after the EMR. These results are similar to the pathological findings after CbFAS alone of previous animal studies [11]. In addition, there were no complications during the follow-up period, even when the deep tissue damage extended into the adventitia in the acute phase in the present study. In the previous porcine animal studies of RFA for the mucosal defect after an ER, two delayed perforations (11\%) occurred, and they concluded that single-step treatment with EMR and RFA was not recommended in the clinical practice [12]. A key feature of cryoablation is that it does not disrupt the extracellular matrix, unlike RFA [13], and may not lead to complications such as perforation, despite the tissue damage it causes.

We used an ablation duration of 8 seconds in this study, although recent clinical trials and reports have used ablation time of 8 to 12 seconds $[5-7,14]$. This is because pigs are more prone to stenosis than humans after endoscopic resection or ablation $[15,16]$. Moreover, previous reports of cryoablation in pigs and humans have shown that pigs have deeper and more severe injuries than do humans [17]. Based on these previous reports, the ablation time was restricted to 8 seconds.

There are four major ablation devices that can be used for esophageal neoplastic lesions in the clinical scenario: photodynamic therapy (PDT), argon plasma coagulation (APC), RFA, and cryoablation. PDT has shown favorable antitumor effects. However, it requires the intravenous administration of a photosensitizer drug and has additional side effects, such as phototoxicity, esophageal stricture, and severe pain [18]. APC is a noncontact electrocoagulation technique that uses argon gas for ablation, and it can only treat small residual or recurrent areas following EMR, ESD, or RFA [19]. Moreover, in spray cryoablation, cryogenic fluids are applied over the esophageal mucosa by using a spray catheter, it requires large equipment, and it is difficult to control to target specific areas in superficial lesions. Currently, RFA has the highest amount of supporting evidence for clinical application, and has been recommended as the standard ablation technique, especially for Barrett's neoplasia, by several national and international associations for endoscopy $[20,21]$. However, there are some obstacles must be overcome in RFA for ESCC with regard to the durability of the treatment effect [22]. CbFAS is a simple and effective procedure for superficial ESCC as well as Barret's neoplasia, and it is associated with less postprocedural pain than is RFA [9]. Therefore, we believe this to be a key advantage of CbFAS and evaluated the feasibility of the procedure both before and after ER, which constitute the most important unmet clinical needs in the management for patients with ESCC. According to the results of this study, we can carefully attempt to evaluate the safety and efficacy of this combination strategy for lesions with suspected deep invasion or large lesions that are at risk of esophageal stenosis in a clinical trial. 
This study has some limitations. We only used small numbers of animal model and could not evaluate the antitumor effect for neoplastic lesions. While both tissue thickness and fragility differ between humans and animal models, we will evaluate the direct effects as well as safety of the combination of CbFAS with ER for neoplastic lesions in a carefully designed clinical trial.

Combination treatment with ER and CbFAS is technically feasible and did not differ from CbFAS alone with regard to tissue damage. Thus, this combination treatment strategy offers a therapeutic option for the management of metachronous and large SESCCs.

\section{List Of Abbreviations}

APC argon plasma coagulation

CbFAS cryoballoon focal ablation system

EMR endoscopic mucosal resection

ER endoscopic Resection

ESD endoscopic submucosal dissection

RFA radiofrequency ablation

SESCC superficial esophageal squamous cell carcinoma

PDT photodynamic therapy

\section{Declarations}

\section{Ethics approval:}

The study protocol was approved by the Animal Experiment Committee in National Cancer Center Japan, and all animal experiments were conducted in accordance with the relevant guidelines and recommendations pertaining to research on animals (K18-024, 2018/12/27).

\section{Availability of data and materials:}

All data generated or analyzed during this study are included in this published article.

\section{Consent to Publication statement:}

Not Applicable 


\section{Competing interests:}

TY received grants for this study from HOYA Pentax Medical Japan.

$H S, Y Y, N T, H H, K T, T K, T F, T A$, and SF declare no potential conflict of interest.

\section{Funding:}

The authors thank Pentax Medical, Redwood City, CA, USA for the provision of resources. This study was partially supported by the National Cancer Center Research and Development Fund (grant no. 29-A-10)

\section{Author contribution statement}

Conceptualization: TY, YY, and HS; Methodology: TY, SF, YY, NT, HH, KT, TF, and HS; Writing - original draft preparation: HS; Writing - review and editing: TY, SF, YY, TK, and TA. All authors reviewed and approved the final manuscript for submission.

\section{References}

1. Fujishiro M, Yahagi N, Kakushima N, Kodashima S, Muraki Y, Ono S, et al. Endoscopic submucosal dissection of esophageal squamous cell neoplasms. Clin Gastroenterol Hepatol. 2006;4:688-94.

2. Katada C, Yokoyama T, Yano T, Kaneko K, Oda I, Shimizu Y, et al. Alcohol consumption and multiple dysplastic lesions increase risk of squamous cell carcinoma in the esophagus, head, and neck. Gastroenterology. 2016;151:860-9.

3. Mizuta H, Nishimori I, Kuratani Y, Higashidani Y, Kohsaki T, Onishi S. Predictive factors for esophageal stenosis after endoscopic submucosal dissection for superficial esophageal cancer. Dis Esophagus 2009;22:626-31

4. Ono S, Fujishiro M, Niimi K, Goto O, Kodashima S, Yamamichi N, et al. Predictors of postoperative stricture after esophageal endoscopic submucosal dissection for superficial squamous cell neoplasms. Endoscopy. 2009;41:661-5.

5. Schölvinck DW, Künzli HT, Kestens C, Siersema PD, Vleggaar FP, Canto MI, et al. Treatment of Barrett's esophagus with a novel focal cryoablation device: a safety and feasibility study. Endoscopy. 2015;47:1106-12.

6. Künzli HT, Schölvinck DW, Meijer SL, Seldenrijk KA, Bergman JG, Weusten BL. Efficacy of the CryoBalloon Focal Ablation System for the eradication of dysplastic Barrett's esophagus islands. Endoscopy. 2017;49:169-75.

7. Ke Y, van Munster SN, Xue L, He S, Zhang Y, Dou L, et al. Prospective study of endoscopic focal cryoballoon ablation for esophageal squamous cell neoplasia in China. Gastrointest Endosc. 2019;90:204-12. 
8. Baust JG, Gage AA, Johansen TB, Baust JM. Mechanisms of cryoablation: clinical consequences on malignant tumors. 2014;68:1-1.

9. van Munster SN, Overwater A, Haidry R, Bisschops R, Bergman JJ, Weusten BL. Focal cryoballoon versus radiofrequency ablation of dysplastic Barrett's esophagus: impact on treatment response and postprocedural pain. Gastrointest Endosc. 2018;88:795-803.

10. Solomon SS, Kothari S, Smallfield GB, Inamdar S, Stein P, Rodriguez VA, et al. Liquid nitrogen spray cryotherapy is associated with less postprocedural pain than radiofrequency ablation in Barrett's esophagus. J Clin Gastroenterol. 2019;53:e84-90.

11. Schöolvinck DW, Friedland S, Triadafilopoulos G, Valli T, van Berge Henegouwen MI, et al. Balloonbased esophageal cryoablation with a novel focal ablation device: dose-finding and safety in porcine and human models. Dis Esophagus. 2017;30:1-8.

12. Herrero LA, van Vilsteren FG, Visser M, Meijer SL, van Berge Henegouwen MI, Bergman JJ, et al. Simultaneous use of endoscopic resection and radiofrequency ablation is not safe in an esophageal porcine model. Dis Esophagus. 2015;28:25-31.

13. Evonich RF 3rd, Nori DM, Haines DE. A randomized trial comparing effects of radiofrequency and cryoablation on the structural integrity of esophageal tissue. J Interv Card Electrophysiol. 2007;19:7783.

14. Canto MI, Abrams JA, Künzli HT, Weusten B, Komatsu Y, Jobe BA, et al. Nitrous oxide cryotherapy for treatment of esophageal squamous cell neoplasia: initial multicenter international experience with a novel portable cryoballoon ablation system (with video). Gastrointest Endosc. 2018;87:574-81.

15. Kamler JP, Borsatto R, Binmoeller KF. Circumferential endoscopic mucosal resection in the swine esophagus assisted by a cap attachment. Gastrointest Endosc. 2002;55:923-8.

16. Schölvinck DW, Alvarez Herrero L, Visser M, Bergman JJ, Weusten BL. Effects of Lugol staining on stenosis formation induced by radiofrequency ablation of esophageal squamous epithelium: a study in a porcine model. Dis Esophagus. 2015;28:603-11.

17. Louie BE, Hofstetter W, Triadafilopoulos G, Weusten BL. Evaluation of a novel cryoballoon swipe ablation system in bench, porcine, and human esophagus models. Dis Esophagus. 2018;31.

18. Wu, H., Minamide, T. and Yano, T. Role of photodynamic therapy in the treatment of esophageal cancer. Dig Endosc. 2019;31:508-16.

19. di Pietro M, Canto MI, Fitzgerald RC. Endoscopic management of early adenocarcinoma and squamous cell carcinoma of the esophagus: screening, diagnosis, and therapy. Gastroenterology. 2018;154:421-36

20. Weusten B, Bisschops R, Coron E, Dinis-Ribeiro M, Dumonceau JM, Esteban JM, et al. Endoscopic management of Barrett's esophagus: European Society of Gastrointestinal Endoscopy (ESGE) Position Statement. Endoscopy. 2017;49:191-98

21. Fitzgerald RC, di Pietro M, Ragunath K, Ang Y, Kang JY, Watson P, et al. British Society of Gastroenterology guidelines on the diagnosis and management of Barrett's oesophagus. Gut. 2014;63:7-42. 
22. Yu X, van Munster SN, Zhang Y, Xue L, Fleischer DE, Weusten BLAM, et al. Durability of radiofrequency ablation for treatment of esophageal squamous cell neoplasia: 5-year follow-up of a treated cohort in China. Endosc. 2019;89:736-48.

\section{Figures}

Figure 1

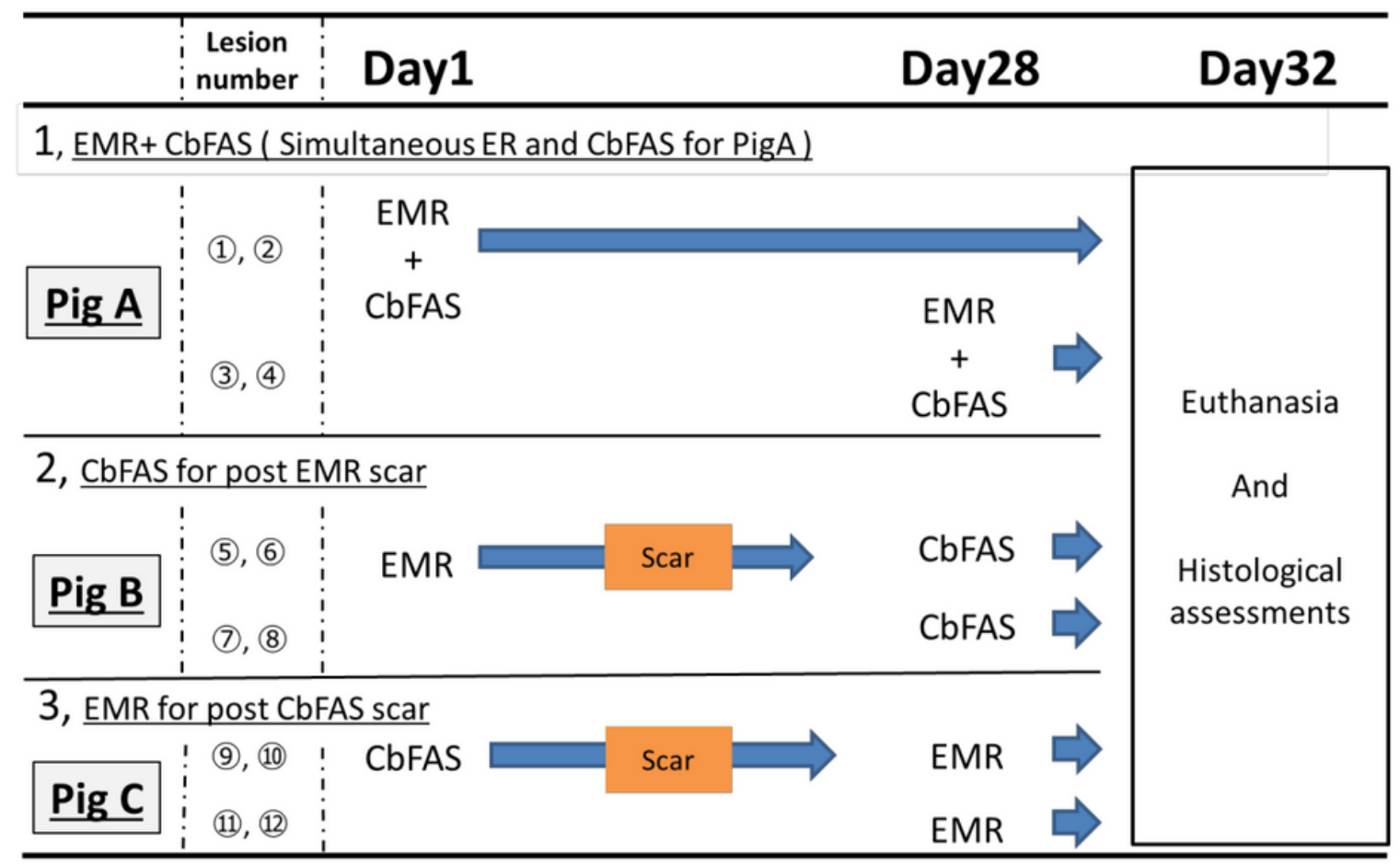

Figure 1

Study flowchart 


\section{Figure 2}

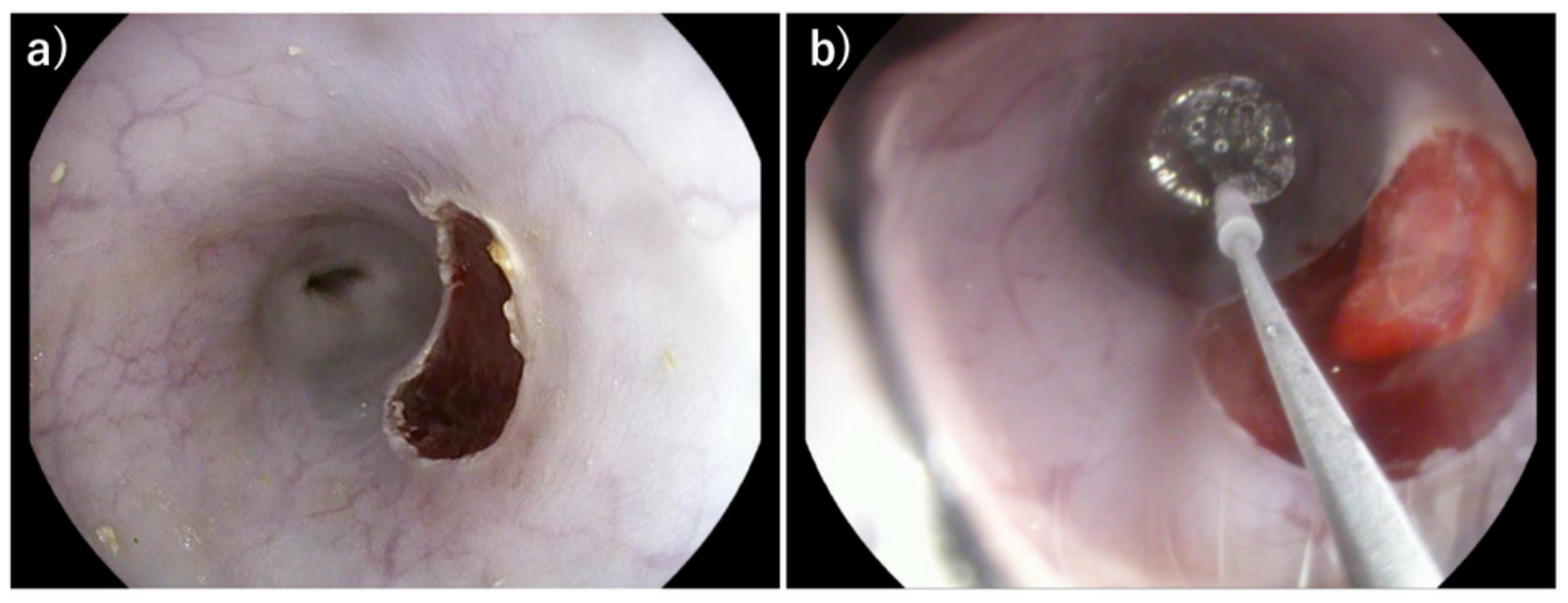

Figure 2

a) Mucosal defects were seen after endoscopic mucosal resection using the Cap method at the right wall of the esophagus. b) The Cryoballoon focal ablation system was applied at the mucosal defect site

Figure 3

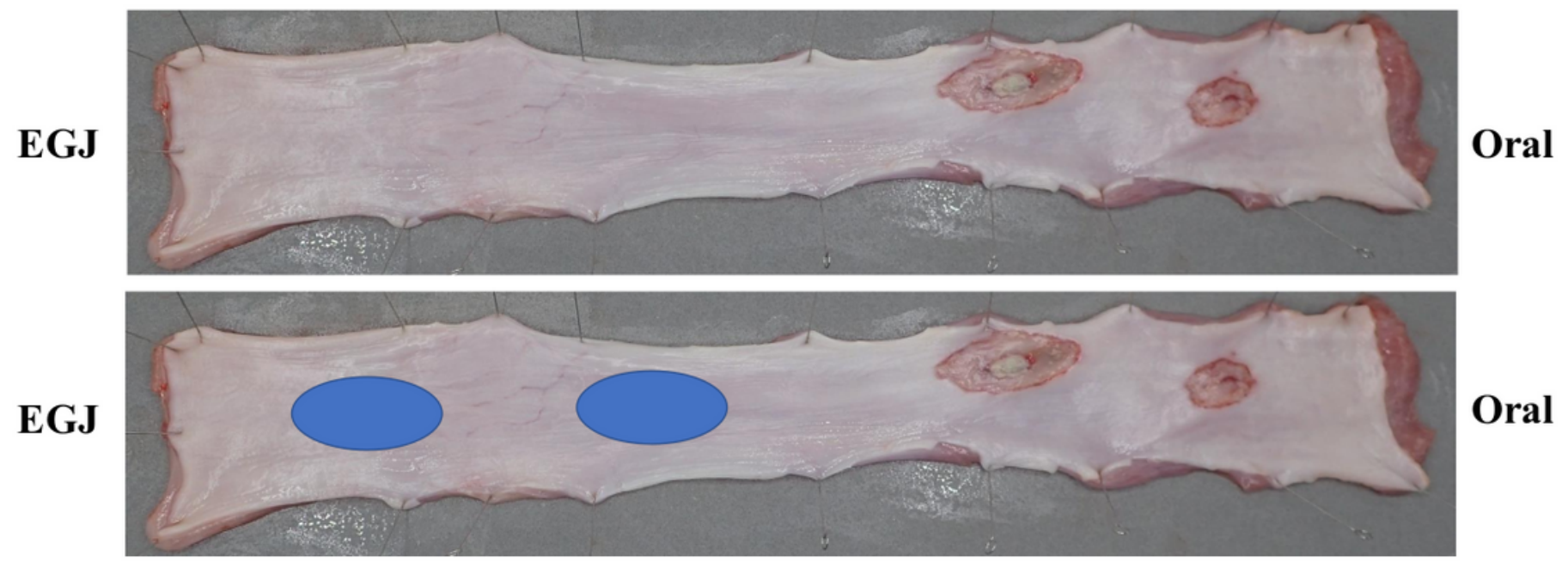




\section{Figure 3}

Esophagus after excision The treatment (Simultaneous use of endoscopic mucosal resection and Cryoballoon focal ablation system) was performed in the blue circle position, but there was no obvious scarring or stenosis.

\section{Figure 4}
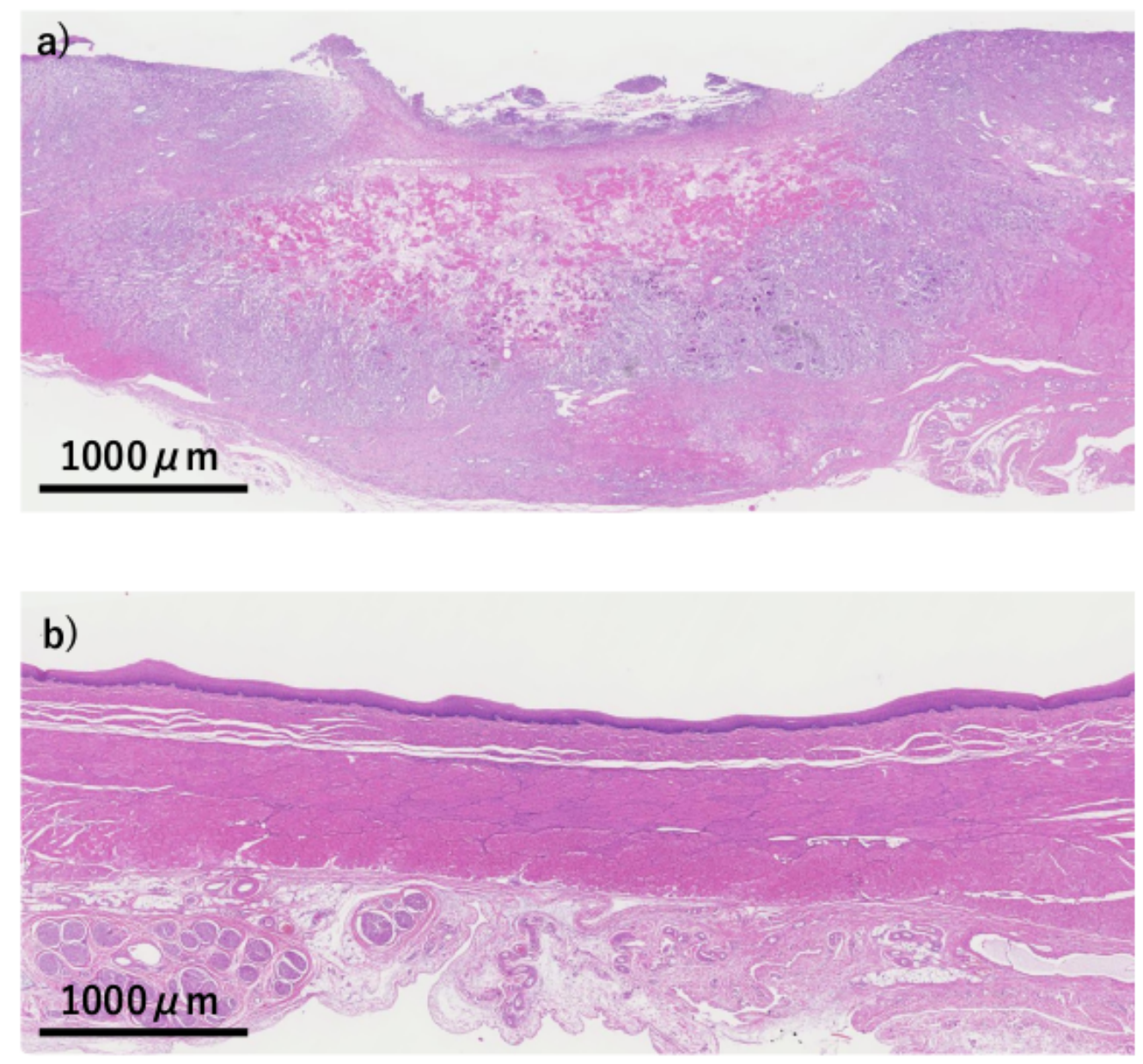

\section{Figure 4}

Histological effects at different time frames after esophageal focal cryoablation a) The Cryoballoon focal ablation system + endoscopic mucosal resection resulted in necrosis throughout the entire esophageal wall at 4 days after treatment. b) The treated areas showed only mild fibrosis 32 days after the treatment.

\section{Supplementary Files}

This is a list of supplementary files associated with this preprint. Click to download.

- supple1.wmv 